\title{
Blood Flow Visualization by Means of Laser Speckle-Contrast Measurements under the Conditions of Nonergodicity
}

\author{
A. Yu. Sdobnov ${ }^{a}$ * , V. V. Kalchenko ${ }^{b}$, A. V. Bykov ${ }^{a}$, A. P. Popov ${ }^{a}$, \\ G. Molodij ${ }^{b}$, and I. V. Meglinski ${ }^{a, c, d, e, f, * *}$ \\ ${ }^{a}$ University of Oulu, Optoelectronics and Measurement Techniques Laboratory, Oulu, 90570 Finland \\ ${ }^{b}$ Weizmann Institute of Science, Department of Veterinary Resources, Rehovot, 76100 Israel \\ ${ }^{c}$ Interdisciplinary Laboratory for Biophotonics, National Research Tomsk State University, \\ Tomsk, 634050 Russia \\ ${ }^{d}$ Institute of Engineering Physics for Biomedicine, National Research Nuclear University (MEPhI), \\ Moscow, 115409 Russia \\ ${ }^{e}$ Aston Institute of Materials Research, School of Engineering and Applied Science, Aston University, \\ Birmingham, B4 7ET, UK \\ ${ }^{f}$ School of Life and Health Sciences, Aston University, B4 7ET Birmingham, UK \\ *e-mail: anton.sdobnov@oulu.fi \\ **e-mail:i.meglinski@aston.ac.uk
}

\begin{abstract}
The influence of stationary structural inclusions in inhomogeneous strongly scattering media such as biotissues on the results of speckle-contrast measurements using temporal and spatial methods of image processing by means of laser speckle-contrast imaging is investigated. The limits of applicability of the method of laser speckle-contrast imaging are established in detail for the case in which ergodicity conditions are not fulfilled. Based on model experiments, it is demonstrated that an increase in the number of stationary scatterers relative to that of the dynamic ones in the sampling volume introduces substantial error into the results of spatial and temporal processing of speckle images for given exposure time of the detector. At the same time, analysis of spatial and temporal speckle contrasts, the values of the coefficient of speckle dynamics, along with the results of Monte-Carlo simulation of the sampling volume, revealed that the presence of a relatively thin, up to $30 \%$ of entire volume, static layer does not introduce considerable changes into the results of measurements by the method of laser speckle-contrast imaging. The exposure time of the camera, along with the number of frames used for image processing, can be varied and chosen individually for each experiment. The developed algorithms of spatial and temporal processing of images obtained by the method of laser speckle-contrast imaging were tested in the experiments on transcranial visualization of the cerebral blood flow of a mouse.
\end{abstract}

Keywords: scattering, laser radiation, laser speckle-contrast imaging, ergodicity, noninvasive imaging, blood flow, brain

\section{INTRODUCTION}

The method of Laser Speckle-Contrast Imaging (LSCI) is relatively simple and promising for in vivo investigation of morphological and functional changes in the blood flow and perfusion in biological tissues [1]. The method is based on statistical analysis of interference speckle patterns formed on the surface of a randomly inhomogeneous turbid medium as a result of scattering of laser radiation. The LSCI is successfully used for visualization of blood and lymph flows in tumor tissues [2-4], estimation of the influence of allergens and optical clearing agents on skin microcirculation $[5,6]$, monitoring of cerebral blood flow [7,
8], monitoring of skin perfusion [9], etc. The advantages of LSCI include the possibility of visualization of the blood flow and perfusion in biological tissues in real time, the possibility of conducting noninvasive measurements, as well as a relatively low cost of the experimental setup.

Classical theory of speckle formation was described in detail in [10]. In fact, laser speckle represents a random interference pattern appearing as a result of interaction of coherent light with a scattering surface and/or a turbid inhomogeneous medium. Under the experimental observation conditions, an image formed in each pixel of a photodetector (a CCD or CMOS camera) represents a superposition of multiple 
amplitude scattering functions each of which is formed by different scattering points of the medium under observation. Hence, the scattering functions are characterized by different phases, which leads to the appearance of a random interference pattern that is referred to as the speckle or the speckle pattern [11]. Changes in local dynamics in the object under observation (motion of scattering particles) results in fluctuations of the speckle pattern. Detection of such a speckle pattern by a camera with a finite exposure time leads to blurring of the obtained speckle image in the regions corresponding to motion of scattering particles. Moreover, the more intense the motion of particles in the object under observation, the more blurred the speckle image obtained by a camera. Statistical analysis of the speckle pattern allows estimating the speed of particles in the medium [11].

To obtain such an estimate, speckle-contrast parameter $K$ was introduced in [12]:

$$
K=\frac{\sigma}{\langle I\rangle},
$$

where $\langle I\rangle$ is the average intensity of the speckle pattern, and $\sigma$ is the standard deviation of intensity.

Theoretically, $\langle I\rangle$ and $\sigma$ are equal to each other in the case of an ideally formed speckle pattern. This means that the speckle contrast must be equal 1 in this case. Nevertheless, the properties of the optical system, the properties of the object under investigation, and coherence of the laser source influence formation of the speckle pattern. As a result, in practice, the speckle contrast attains values between 0 and 1 .

Using the theory of correlation functions, an interrelation of speckle contrast, camera exposure time, and speckle correlation time was demonstrated in [12]. It was suggested in [13] that temporal fluctuations of speckle-pattern intensity caused by variation of the electric field due to light scattering by moving particles can be related to the real velocity of these particles. A quantitative estimate of the temporal fluctuations can be obtained using a normalized autocorrelation function of the electric field [14]:

$$
g_{1}(\tau)=\frac{\left\langle E(t) E^{*}(t+\tau)\right\rangle}{\left\langle E(t) E^{*}(t)\right\rangle},
$$

where $E(t)$ is the electric field of the detected light, $E^{*}(t)$ is the complex conjugate of the electric field at time $t$, and $\tau$ is the autocorrelation delay time. For Gaussian random optical fields, autocorrelation (2) is related to the autocorrelation function of the scattered light intensity:

$$
g_{2}(\tau)=\frac{\langle I(t) I(t+\tau)\rangle}{\left\langle I(t)^{2}\right\rangle}
$$

Second-order autocorrelation function (3) can be expressed in terms of first-order autocorrelation function (2) through the Ziegert relationship [15]:

$$
g_{2}(\tau)=1+\beta\left|g_{1}(\tau)\right|^{2},
$$

where $\beta(0 \leq \beta \leq 1)$ is the coefficient of multiplicative decrease of the speckle contrast related to such factors as polarization, effects of coherence, and mismatch between pixel size of the camera and the average size of a speckle [16].

Hence, equation (1) governing the speckle contrast can be presented in the form

$$
K(T)=\left[\frac{2 \beta}{T} \int_{0}^{T}\left|\frac{g_{1}(\tau)}{g_{1}(0)}\right|^{2}\left(1-\frac{\tau}{T}\right) d \tau\right]^{1 / 2},
$$

where $K(T)$ is the speckle-contrast function that depends on the camera exposure time.

In the case of scattering from an ensemble of statistically independent particles, the detected field represents a superposition of a large number of partial statistically independent fields. According to the central limit theorem, statistics of such a field is Gaussian, and Ziegert relationship (3) is real only under these conditions. In addition, the theory described above is based on the assumption that the studied medium is spatially homogeneous, dynamic, and laser radiation scattered from the medium satisfies the ergodicity condition [17, 18]. In practice, biological tissues are strongly inhomogeneous spatially and consist of dynamic inclusions (blood, lymph), along with static components (skin, bones, etc.). A scattering system of this kind does not satisfy the ergodicity condition, which results in the appearance of a systematic error in the LSCI method and, as a consequence, in incorrect interpretation of the obtained data [16, 19-21].

The possibility of applying the LSCI method in the case of violation of the ergodicity condition was analyzed in a number of studies [16, 22-26]. The detected laser radiation scattered in biological tissues can be presented as a sum of dynamic $\left(E_{d}(t)\right)$ and static $\left(E_{s}\right)$ components of the electric field:

$$
E(t)=E_{d}(t)+E_{s} .
$$

Hence, the autocorrelation function of the field can be presented in the form

$$
g_{1}(\tau)=(1-\rho)\left|g_{1 d}(\tau)\right|+\rho,
$$

where $\rho=I_{s} /\left(I_{d}+I_{s}\right)$ is part of the detected light scattered from the static scattering particles.

The intensity autocorrelation function, in turn, takes the form

$$
g_{2}(\tau)=1+\beta\left[(1-\rho)\left|g_{1 d}(\tau)\right|+\rho g_{1}(0)\right]^{2} .
$$

Taking into account equations (6) $-(8)$, the speckle contrast can be expressed in the form

$K(T)=\left[\frac{2 \beta}{T} \int_{0}^{T}\left[\frac{(1-\rho) \mid g_{1 d}(\tau)+\rho g_{1}(0)}{g_{1}(0)}\right]^{2}\left(1-\frac{\tau}{T}\right) d \tau\right]^{1 / 2}$. 
In the case of a fully dynamic medium, equation (9) reduces to equation (5) due to the absence of the static scattering particles $(\rho \rightarrow 0)$. Nevertheless, equation (9) does not reduce to a constant value of the speckle contrast in the case of a static scattering medium, as could be expected from equation (1).

Despite substantial limitations in the applicability of the LSCI method under the conditions of violation of ergodicity, various methods were proposed. In particular, a multi-exposure LSCI [27] and using a slowly rotating diffuser in the optical setup [26] proved to be efficient solutions.

It is important to note that the terms "ergodicity" and "nonergodicity" in the present work are used under the conditions of a finite camera exposure time. In general, ergodicity assumes that the observation time of an object is infinite. However, under experimental conditions, the time of each individual measurement is limited by the camera exposure time, and obtained image represents an averaged signal. Moreover, in most cases, the camera exposure time is much longer than the relaxation time of the dynamic component of the speckle pattern but shorter than the relaxation time of the static one. Hence, the studied medium can be classified as being ergodic or nonergodic depending on the camera exposure time. For example, a very slow flow represents an ergodic process in a general sense, because the temporal and spatial statistics are identical in the case of an infinite observation time. Nevertheless, when recorded by a camera with a very short exposure time, this process will be classified as a nonergodic one [23].

The present work aims at investigation of the applicability boundaries of the LSCI in the case when the ergodicity condition is not fulfilled, as well as at estimating optimal parameters for conducting measurements with live objects. In this regard, we investigated the influence of stationary structural inclusions in an inhomogeneous strongly scattering media, such as biological tissues, on the results of the speckle-contrast measurements using temporal and spatial methods of image processing by the LSCI method. As part of this study, we tested the LSCI method in the experiments on transcranial in vivo visualization of the brain of a mouse.

\section{MATERIALS AND METHODS}

The optical layout of the experimental setup used in the present work is illustrated in Fig. 1. A laser diode with an output wavelength of $655 \mathrm{~nm}$ and power of $13 \mathrm{~mW}$ (RLD650-13-3, Roithner Lasertechnik $\mathrm{GmbH}$, Austria) was used as a source of radiation in the experiment with a phantom. In the experiment with a mouse, we used a laser diode operating at $808 \mathrm{~nm}$ and delivering $3 \mathrm{~mW}$ of power (LDM808/3LJ, Roithner Lasertechnik GmbH, Austria). A diffuser (ED1-C20, Thorlabs, USA) was used to obtain uni-

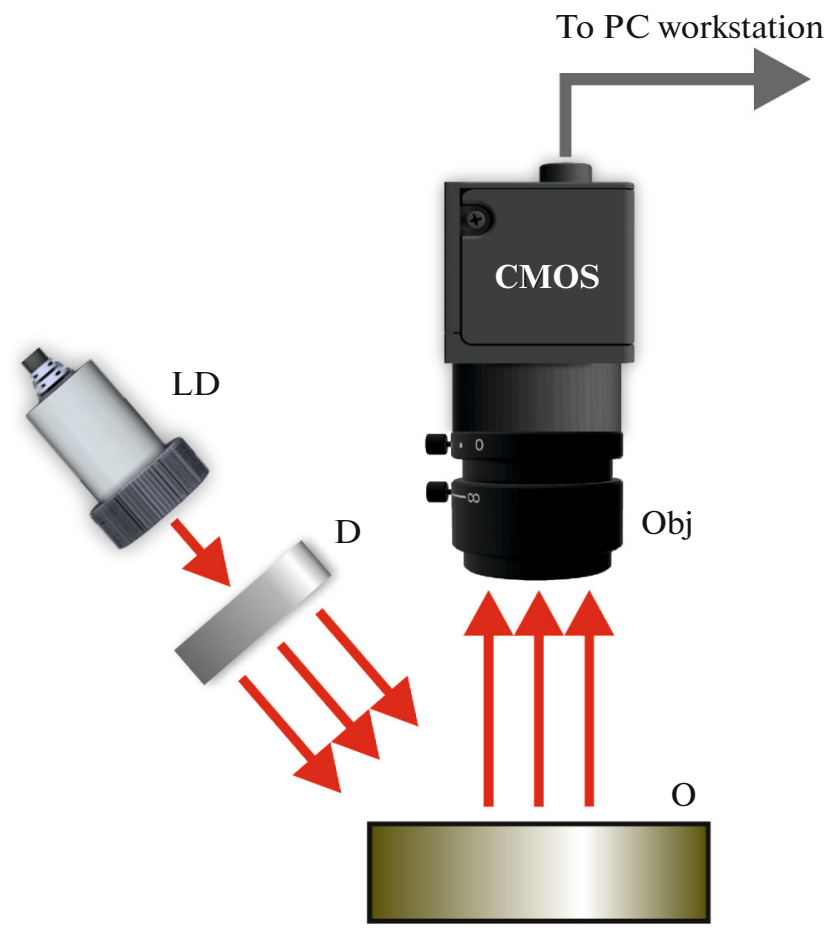

Fig. 1. Optical layout of the experimental setup for LSCI. CMOS-imaging camera; LD-laser diode; D-diffuser; Obj-objective lens; O_object.

form distribution of the laser radiation over the studied object. A CMOS camera (DCC3240M, $1280 \times 1024$, pixel size $6.7 \mu \mathrm{m}$, Thorlabs, USA) in combination with a 12-mm F1.4 objective lens (Kenko Tokina Co., Ltd, Japan) was used for speckle-pattern acquisition. The obtained images were processed by means of a specially developed algorithm based on MATLABr2019b software.

The spatial algorithm of analysis of speckle statistics is the most widely used method for processing the LSCI images [28]. In this algorithm, speckle statistics is calculated within a window sliding along a raw speckle image. As a rule, a window with a size of $5 \times 5$ or $7 \times 7$ pixels is used for obtaining an optimal result. The major drawback of the spatial algorithm consists in the loss of spatial resolution determined by the size of the sliding window. To resolve this problem, an alternative algorithm of temporal analysis of speckle statistics was proposed in [29]. In this algorithm, a series of consecutive speckle images is obtained. After that, speckle statistics is calculated from one image to another for each individual pixel of the series. In this way, spatial resolution can be preserved at the expense of losing the temporal one. In practice, a combination of both methods with parameters chosen depending on the problem being solved is usually used [30].

In the case of light scattering from dynamic particles, the temporal and spatial algorithms yield identical results, because the ergodicity condition is ful- 
Table 1. Optical parameters of the sticky tape and intralipid at $655 \mathrm{~nm} . \mu_{\mathrm{s}}$-scattering coefficient, $\mu_{\mathrm{a}}$-absorption coefficient, $g$-anisotropy factor, $n$-refractive index, $\mu_{s}^{\prime}-$ reduced scattering coefficient.

\begin{tabular}{c|c|c|c|c}
\hline \multicolumn{5}{c}{ Sticky tape } \\
\hline$\mu_{\mathrm{s}}, \mathrm{mm}^{-1}$ & $\mu_{\mathrm{a}}, \mathrm{mm}^{-1}$ & $g$ & $n$ & $\mu_{s}^{\prime}, \mathrm{mm}^{-1}$ \\
\hline 36.38 & 0 & 0.946 & 1.6 & 1.96 \\
\hline \multicolumn{5}{c}{ Intralipid, 3\% } \\
\hline$\mu_{s}$ & $\mu_{a}$ & $g$ & $n$ & $\mu_{s}^{\prime}$ \\
\hline 11.8 & 0.02 & 0.55 & 1.33 & 5.31 \\
\hline
\end{tabular}

filled. In the case of scattering from static scatterers, the results of spatial and temporal processing of speckle images turn out to be different. In particular, theoretical value of the spatial speckle contrast for an ideally formed static speckle pattern is equal to 1 , because spatial fluctuations of intensity (dark and bright speckles) attain their minimum and maximum values of intensity, respectively. In this case, the average intensity equals the standard deviation of intensity. The temporal speckle contrast should be equal to 0 , because standard deviation in each pixel attains the value of 0 for a static speckle pattern. In practice, it seems impossible to create ideal conditions. Therefore, the speckle contrast attains values between 0 and 1. Nevertheless, the described difference between the temporal and spatial speckle contrasts in the case of presence of static scatterers can be considerable and can result in a systematic error in the interpretation of the obtained results.

In our previous work [24], we introduced a coefficient of speckle dynamics (CSD) that allows estimating the influence of the nonergodic component on the results of measurements by means of the LSCI:

$$
C S D=\frac{2 K_{t}}{K_{s}+K_{t}}
$$

where $K_{t}$ is the speckle contrast calculated by using the temporal algorithm, while $K_{s}$ is the speckle contrast calculated by using the spatial one. Theoretically, this coefficient attains the value of 1 for a medium dynamically scattering light and 0 for a turbid medium consisting of static scatterers. Nevertheless, in practice, the CSD also does not reach minimum and maximum theoretical values since $K_{t}$ and $K_{s}$ attain values between 0 and 1.

A rectangular phantom with a hole $(1 \mathrm{~cm}$ in diameter) on the surface was fabricated from silicone for model experiment [31]. A 3-\% intralipid solution was used as a system of dynamic scatterers. A nontransparent sticky scotch tape with a thickness of $55 \pm 2 \mu \mathrm{m}$ was used for mimicking the static layers. A total of 49 consecutive frames obtained with a camera exposure time of $10 \mathrm{~ms}$ were acquired for calculation of the coefficient of speckle dynamics of the phantom. These frames were used for calculation of the temporal speckle-contrast image. In addition, 49 spatial speckle-contrast images were calculated with a sliding-window size of $7 \times 7$ pixels. After that, 49 images of the coefficient of speckle dynamics were calculated that were subsequently averaged to form a final image. The spatial speckle-contrast images were also averaged to form one final image.

An 8-week-old CD1 female mouse supplied by Envigo (UK) was used for transcranial visualization of the brain of a mouse. An intraabdominal injection of $10 \mathrm{mg} / 100 \mathrm{mg} / \mathrm{kg}$ of ketamine (Vetoquinol, France) was used to anesthetize the mouse. After introduction of the anesthetic, skin was removed in the forehead, temporal, crown, and back of the head areas. The studied area of the head was moisturized with a physiological solution. After that, the mouse was placed on a warming pad to maintain her body temperature at $37^{\circ} \mathrm{C}$. The total duration of the measurements did not exceed $1.5 \mathrm{~h}$, after which the animal was euthanized by an overdose of barbiturate. To calculate the images of the coefficient of speckle dynamics of the mouse head, a sequence of frames of different size was obtained with an exposure time of $10 \mathrm{~ms}$. After that, these frames were used to calculate the temporal specklecontrast image using the same principle as in the experiment with the phantom.

\section{RESULTS AND DISCUSSION}

To demonstrate the influence of an increase in the thickness of layer of static scatterers on the results of measurements conducted by the LSCI method, we carried out the following experiment. The hole in the phantom was filled with an intralipid solution. The surface of the phantom was covered by layers of a nontransparent sticky tape, layer by layer. Addition of each sticky-tape layer can be considered as an increase in the thickness of the layer of static scatterers above the medium dynamically scattering light (an intralipid solution). A total of 16 layers were added in the experiment. Their combined thickness was equal to $880 \mu \mathrm{m}$. Optical properties of the intralipid solution [32] and the sticky tape used for modeling are presented in the table 1. Optical properties of the sticky tape were measured experimentally using an integrating sphere [33].

Examples of spatial and temporal speckle-contrast images, along with the images of the coefficient of speckle dynamics, calculated for different number of layers of static scatterers on phantom surface are presented in Fig. 2. Figure 3 illustrates the average values of the temporal and spatial speckle contrast, along with the average value of the coefficient of speckle dynamics, calculated within the area marked by a green square in Fig. 2 for the discussed experiment as functions of the number of static layers. Also, the corresponding interpolation curves are shown. 


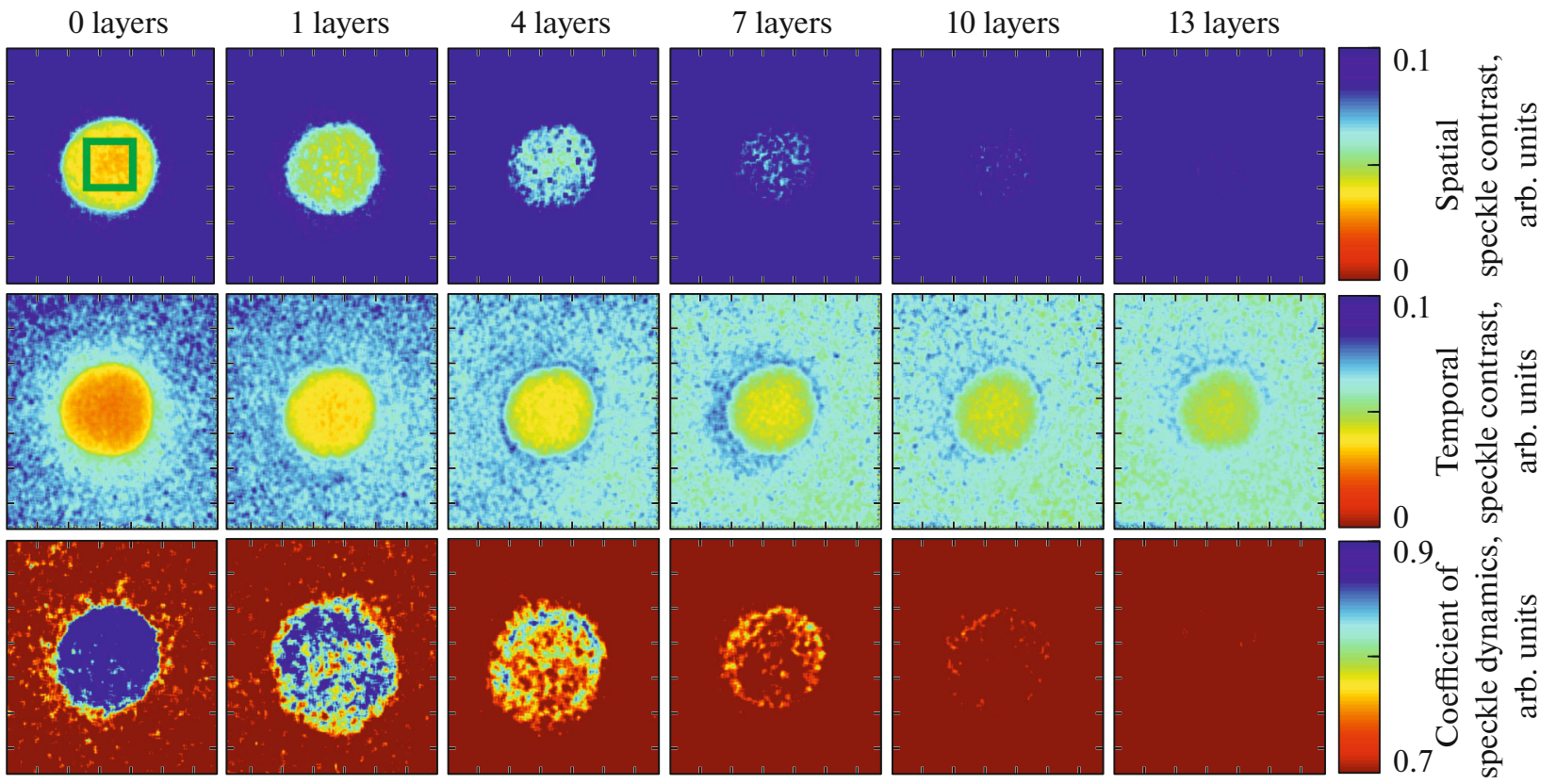

Fig. 2. Spatial speckle-contrast images (upper row), temporal speckle-contrast images, along with images of the coefficient of speckle dynamics, calculated for the experiment with a phantom filled with an intralipid solution without static layer (0 layers) on the surface and with 1, 4, 7, 10, and 13 static layers on the surface. The length of the scale bar is $5 \mathrm{~mm}$.

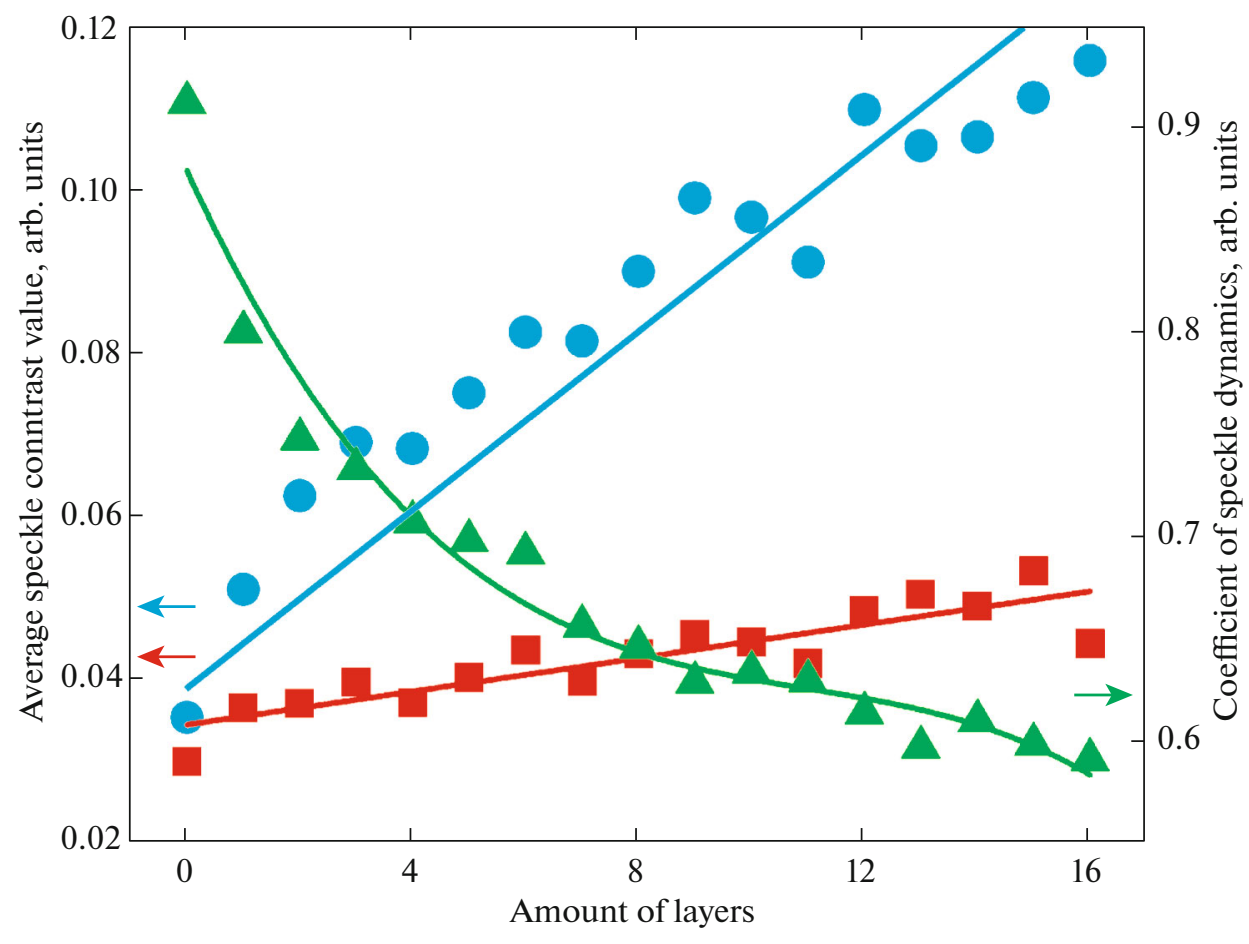

Fig. 3. (Color online) Average value of the spatial speckle contrast (blue circles), average value of the temporal speckle contrast (red squares), and average value of the coefficient of speckle dynamics (green triangles) as functions of the number of static layers on phantom surface. Symbols denote experimental values. Solid lines represent the interpolation curves. 
It can be seen from Figs. 2 and 3 that intensity of the Brownian motion of particles of the intralipid solution remains nearly unchanged, while the values of the spatial and temporal speckle contrast increase linearly with successive addition of sticky-tape layers. It can be seen that the values of the spatial speckle contrast increase 4 times faster relative to those of the temporal speckle contrast. The values of the speckle contrast can thus depend not only on the velocity of scattering particles but also on the thickness of the layer of static scatterers above the medium dynamically scattering light. This effect is usually ignored in studies using the LSCI method. Nevertheless, the influence of thickness of the layer of static scatterers on the value of speckle contrast must be taken into account, especially, when conducting measurements with biological objects the blood vessels in which lie at various depths. A different value of the speckle contrast can be a manifestation of not only the blood-flow velocity in the blood vessels but also of their depth, which could, in turn, lead to a systematic error in analysis and interpretation of the experimental data obtained by means of the LSCI. Calculation of the coefficient of speckle dynamics allows distinguishing between changes in the speckle contrast caused by an increase or a decrease in the intensity of motion of scatterers and those caused by the presence of a statically scattering layer above the medium scattering light dynamically [24].

It can also be seen from Figs. 2 and 3 that the spatial and temporal speckle contrasts attain close to each other values in the absence of the static scattering layer above the intralipid solution, which indicates that the system under consideration satisfies the ergodicity condition. The difference between the spatial and temporal speckle contrasts increases with addition of static layers. Simultaneously, the value of the coefficient of speckle dynamics decreases, which indicates the growth of violations of the ergodicity condition. These violations cause the appearance of a systematic error in the LSCI method and must be taken into consideration accordingly when conducting measurements with biological objects. It can be seen from Fig. 3 that the value of the temporal speckle contrast is influenced less by an increase in the static-layer thickness. Moreover, the region filled with intralipid remains visible in the course of temporal processing even in the presence of a large number of static scattering layers. Hence, the quantitative estimate of the blood-flow velocity in the blood vessels can strongly depend on the chosen method of speckle-image processing. At the same time, the fact that the system under consideration does not comply with the ergodicity condition will not impact qualitative measurements in which it is important to demonstrate only the relative changes in the blood flow or perfusion. It can also be seen from the figures that the value of the coefficient of speckle dynamics decreases inversely proportional to an increase in the fraction of static scatter- ers (the nonergodic component). Presumably, the presence of static layers of small thickness does not introduce substantial error in the estimate of speckle contrast. Hence, the coefficient of speckle dynamics can be used to estimate the influence of depth of a blood vessel on the result of the speckle-contrast measurements.

The Monte-Carlo method [34] was used to estimate the effective sampling volume in the model experiments carried out with phantoms containing various numbers of static layers. The results of simulation of the effective sampling volume for the model experiments conducted with phantoms containing various numbers of static layers are illustrated in Fig. 4. Optical properties presented in the Table 1 correspond to radiation with a wavelength of $655 \mathrm{~nm}$. To estimate independent of wavelength effective sampling volume of a tissue, the data of simulation were plotted as a function of optical density that was calculated as a product of the layer thickness $(D)$ and the reduced scattering coefficient $\left(\mu_{s}^{\prime}\right)$. It can be seen that considerable effective amount of radiation scattered from intralipid is registered by the detector even in the presence of several static layers (up to 7 layers of sticky tape with a total thickness of $385 \mu \mathrm{m}$ ), which allows carrying out more or less accurate measurements using the LSCI.

According to different sources, a typical skull thickness of a mouse at an age of up to 2 months varies from 150 to $310 \mu \mathrm{m}[35,36]$, while infrared radiation is characterized by large penetration depth into biological tissues. The reduced scattering coefficient in the infrared spectral range for a skull of a mouse equals $1.75 \pm 0.05$ [35]. Hence, the presence of a static scattering layer in the form of skull bones does not introduce substantial distortions in the results of the cerebral blood flow measurements of a mouse by means of the LSCI. Examples of spatial and temporal specklecontrast images, along with images of the coefficient of speckle dynamics, calculated using different sequences of the raw speckle images under transcranial in vivo visualization of the cerebral blood flow of a mouse are presented in Fig. 5. As we mentioned above, the skull thickness of a young mouse does not introduce substantial errors in the results of the measurements. It can be clearly seen from Fig. 5 that spatial and temporal speckle-contrast images obtained by processing a sequence of 10 frames are identical for both parts of the brain containing blood vessels (point 2 in Fig. 5) and areas free of blood vessels (point 1 in Fig. 5). The coefficient of speckle dynamics attains high value in the regions of measurements corresponding to the brain, which is indicative of the fact that the ergodicity conditions are either not violated or violations are not significant. Nevertheless, the LSCI is based on statistical measurements. Therefore, it is important taking into account the size of the data sample, which corresponds to the number of consecutive frames used for obtaining the speckle-contrast images 


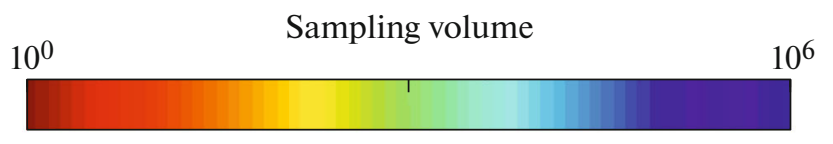

(a)

(b)

(c)

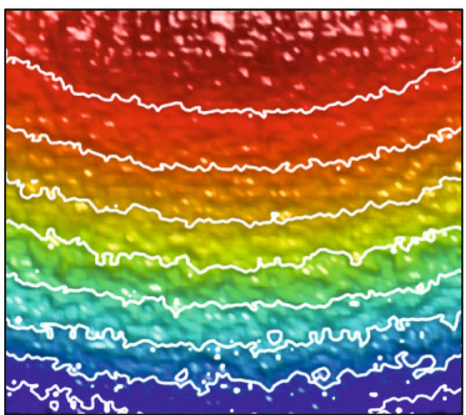

(d)

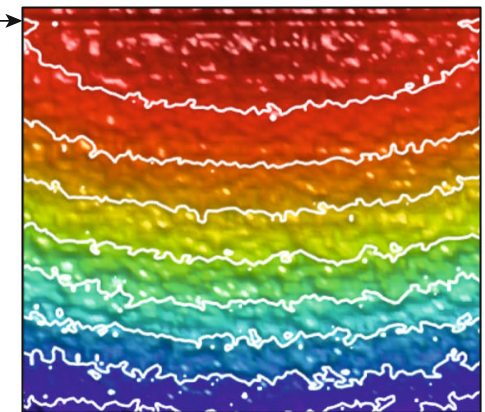

(e)

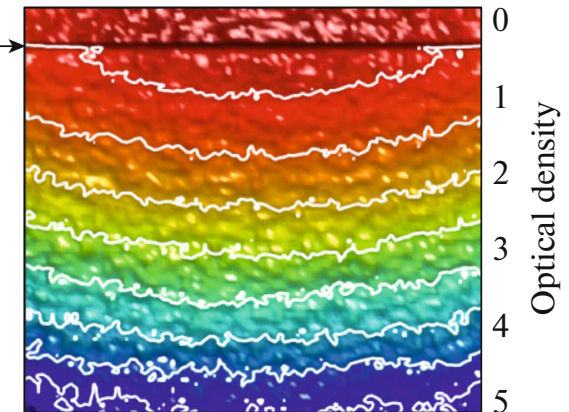

(f)
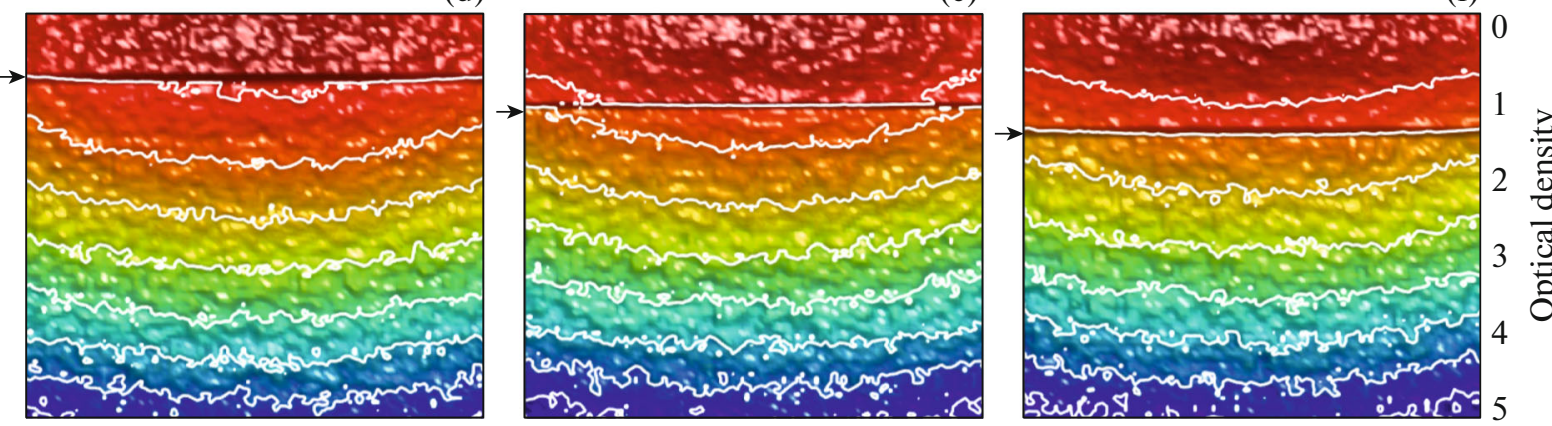

Fig. 4. Monte-Carlo simulation of an effective sampling volume of radiation for intralipid in the absence of a static layer above (a); for intralipid with 1 (b), 4 (c), 7 (d), 10 (e), and 13 (f) static layers above. Black arrow marks position of the static-layer lower boundary.
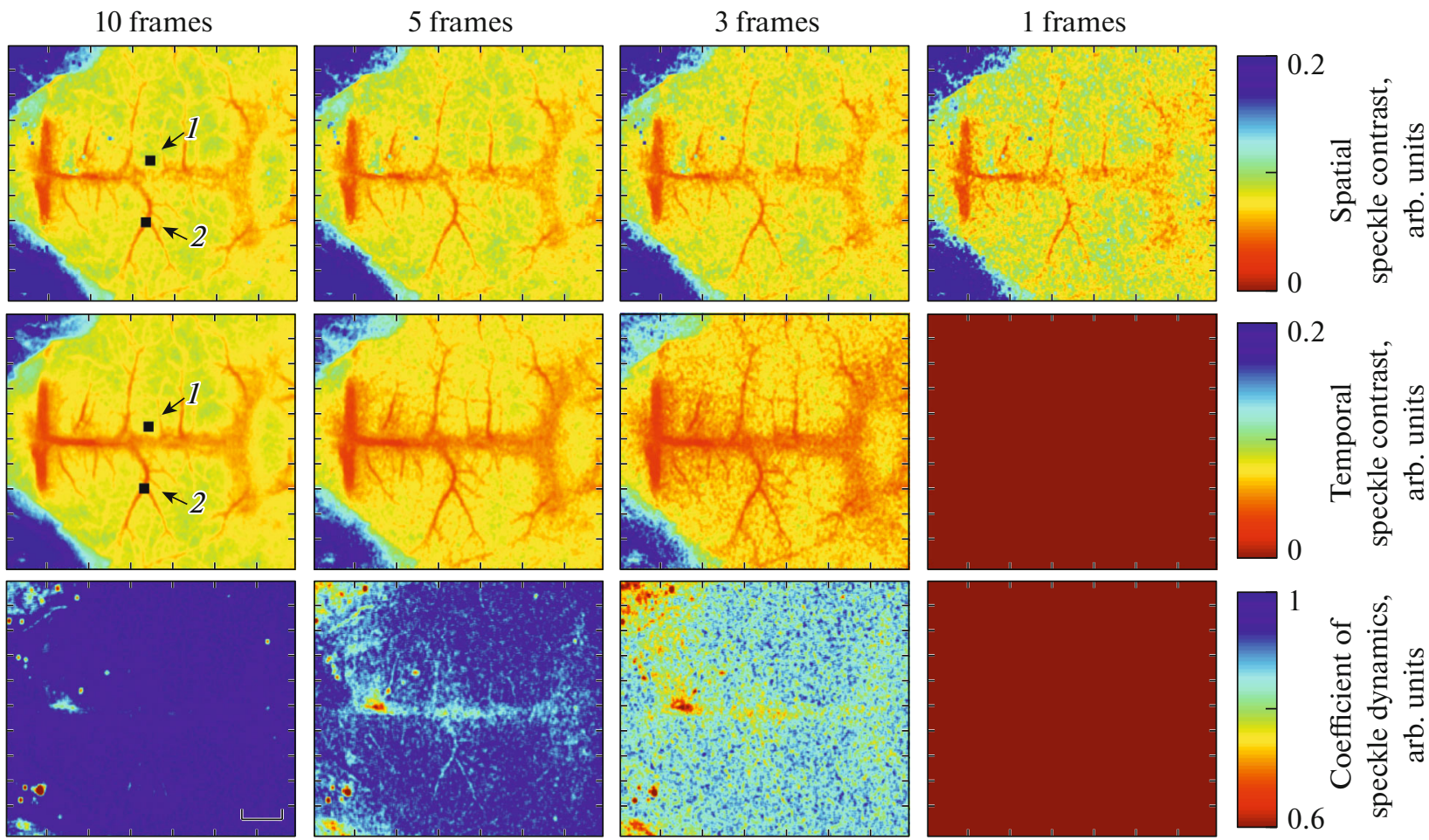

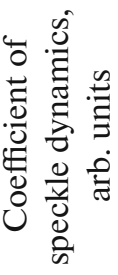

Fig. 5. Spatial speckle-contrast images (upper row), temporal speckle-contrast images, and images of the coefficient of speckle dynamics calculated for the case of transcranial visualization of the brain of a mouse using $10,5,3$, and 1 consecutive speckle images. The length of the scale bar is equal to $1 \mathrm{~mm}$. 
is the case under consideration. It can be seen from Fig. 5 that a reduction in the number of frames strongly influences the result of temporal processing. In particular, the difference between spatial and temporal speckle-contrast images increases for both regions of the brain containing blood flow/blood vessels (point 2 in Fig. 5) and the region in which they are absent (point 1 in Fig. 5), which can also be clearly seen from the image of the coefficient of speckle dynamics. This effect must also be taken into consideration when using the spatiotemporal algorithm of speckle-image processing. It was established in our case, in the experiment carried out with an exposure time of $10 \mathrm{~ms}$, that the minimum sufficient size of the sample of consecutive raw images was equal to 10 frames.

Incorrectly chosen camera exposure time, algorithms of speckle-image processing, the number of frames used for image processing, or the presence of a static scattering layer can substantially impact the results of the measurements conducted by means of the LSCI. Optimal parameters of the LSCI should be chosen depending on whether it is necessary to obtain quantitative or qualitative data, whether it is more important preserving spatial or temporal resolution, etc. The coefficient of speckle dynamics allows estimating the influence of system nonergodicity for given exposure time, which can help choosing optimal parameters for the LSCI measurements. In particular, it was demonstrated in the present work that the skull bones do not introduce substantial distortions from the point of view of fulfillment of the ergodicity condition upon visualization of the cerebral blood flow in the brain of a mouse. Nevertheless, skin must be removed from the region of the measurements in order to minimize the systematic error of the LSCI experiment. In the case of an adult mouse, in addition to skin removal, the procedures of skull thinning by means of a drill [37] or its total removal [38] are applied. Using optical clearing [39] can potentially reduce the influence of the layer of static scatterers of a biological tissue [40] (skin, in particular [40]) on the results of the LSCI, thereby increasing the efficiency of the noninvasive in vivo visualization of the cerebral blood flow.

\section{CONCLUSIONS}

Thus, we investigated the influence of the ergodicity condition of the studied inhomogeneous strongly scattering medium on the results of the LSCI. Using a phantom, it was demonstrated experimentally that an increase in the number of stationary scatterers relative to the number of dynamic ones in the studied volume causes the results of spatial and temporal processing of the speckle images for given exposure time to be different. Analysis of the obtained experimental values of the spatial and temporal speckle contrast, the values of the coefficient of speckle dynamics, along with analysis of the effective sampling volume of radiation simu- lated by means of the Monte-Carlo method, suggest that the presence of a relatively thin, up to $30 \%$ of the total volume, static layer does not introduce considerable changes in the LSCI results. This supposition was confirmed by means of the transcranial in vivo visualization of the cerebral blood flow in the brain of an 8 -week-old mouse. It was demonstrated that violation of the ergodicity condition in the studied object must be taken into account when conducting the measurements. It was also demonstrated that system parameters for the LSCI, such as the camera exposure time, the method of analysis of the speckle images, and the number of frames used for processing, must be chosen individually for each experiment depending on the problem being solved.

\section{FUNDING}

A.S. acknowledges the support of the Finnish Cultural Foundation (grant no. 00180998). I.M. acknowledges partial support of the Academy of Finland (grants nos. 326204 and 325097), NEUROPA Research and Innovation Program Horizon 2020 (project no. 863214), MEPhI Academic Excellence Project no. 232 02.a03.21.0005, and the funding program of D.I. Mendeleev Tomsk State University. A.B. acknowledges the support of the Academy of Finland (grant no. 290596). A.P. acknowledges the support of the Academy of Finland (grant no. 314369) and the INFOTECH project. V.K. acknowledges the support of the Henry Chanoch Krenter Institute for Biomedical Imaging and Genomics (Staff Scientists grant program).

\section{COMPLIANCE WITH ETHICAL STANDARDS}

\section{Statement on the Welfare of Animals}

Animal research has been approved by Weizmann Institute of Science Institutional Animal Care and Use Committee (IACUC). All institutional principles of animal care and use have been met.

\section{Conflict of Interest}

The authors declare that they have no conflict of interest.

\section{REFERENCES}

1. M. J. Leahy, Microcirculation Imaging (Wiley, Chichester, 2012).

2. I. V. Meglinski, V. V. Kalchenko, Y. L. Kuznetsov, B. I. Kuznik, and V. V. Tuchin, Dokl. Phys. 58, 323 (2013). https://doi.org/10.1134/S102833581308003X

3. V. Kalchenko, Y. Kuznetsov, A. Harmelin, and I. V. Meglinski, J. Biomed. Opt. 17, 050502 (2012). https://doi.org/10.1117/1.JBO.17.5.050502

4. V. Kalchenko, Y. L. Kuznetsov, and I. Meglinski, Quantum Electron. 43, 679 (2013).

https://doi.org/10.1070/QE2013v043n07ABEH014953 
5. V. Kalchenko, Y. L. Kuznetsov, D. Preise, I. Meglinski, and A. Harmelin, J. Biomed. Opt. 19, 060502 (2014). https://doi.org/10.1117/1.JBO.19.6.060502

6. V. Kalchenko, I. Meglinski, A. Sdobnov, Y. Kuznetsov, and A. Harmelin, J. Biomed. Opt. 24, 060501 (2019). https://doi.org/10.1117/1.JBO.24.6.060501

7. V. Kalchenko, D. Israeli, Y. L. Kuznetsov, I. Meglinski, and A. Harmelin, J. Biophoton. 8, 897 (2015). https://doi.org/10.1002/jbio.201400140

8. V. Kalchenko, A. Sdobnov, I. Meglinski, Y. Kuznetsov, G. Molodij, and A. Harmelin, Photonics 6, 80 (2019). https://doi.org/10.3390/photonics6030080

9. I. Mizeva, V. Dremin, E. Potapova, E. Zherebtsov, I. Kozlov, and A. Dunaev, IEEE Trans. Biomed. Eng. (2019). https://doi.org/10.1109/TBME.2019.2950323

10. J. W. Goodman, Laser Speckle and Related Phenomena, Ed. by J. C. Dainty, Vol. 9 of Topics in Applied Physics (Springer, Berlin, Heidelberg, 1975), p. 9.

11. J. W. Goodman, Speckle Phenomena in Optics: Theory and Applications (Roberts and Co., Englewood, CO, 2007).

12. A. Fercher and J. Briers, Opt. Commun. 37, 326 (1981). https://doi.org/10.1016/0030-4018(81)90428-4

13. D. Boas and A. G. Yodh, J. Opt. Soc. Am. A 14, 192 (1997). https://doi.org/10.1364/JOSAA.14.000192

14. H. Cummins, Photon Correlation and Light Beating Spectroscopy (Springer Science, New York, 2013), Vol. 3.

15. B. J. Berne and R. Pecora, Dynamic Light Scattering: With Applications to Chemistry, Biology, and Physics (Courier Corp., Chelmsford, MA, 2000).

16. A. B. Parthasarathy, W. J. Tom, A. Gopal, X. Zhang, and A. K. Dunn, Opt. Express 16, 1975 (2008). https://doi.org/10.1364/OE.16.001975

17. W. B. Davenport and W. L. Root, An Introduction to the Theory of Random Signals and Noise (McGraw-Hill, New York, 1958).

18. A. M. Yaglom, An Introduction to the Theory of Stationary Random Functions (Courier Corp., Chelmsford, MA, 2004).

19. P. Zakharov, A. Volker, A. Buck, B. Weber, and F. Scheffold, Opt. Lett. 31, 3465 (2006). https://doi.org/10.1364/OL.31.003465

20. J. G. H. Joosten, E. T. F. Gelade, and P. N. Pusey, Phys. Rev. A 42, 2161 (1990). . https://doi.org/10.1103/PhysRevA.42.2161

21. P. Li, S. Ni, L. Zhang, S. Zeng, and Q. Luo, Opt. Lett. 31, 1824 (2006). https://doi.org/10.1364/OL.31.001824

22. P. Zakharov, Opt. Let. 42, 2299 (2017). https://doi.org/10.1364/OL.42.002299

23. P. N. Pusey and W. van Megen, Phys. A (Amsterdam, Neth.) 157, 705 (1989). https://doi.org/10.1016/0378-4371(89)90063-0
24. A. Sdobnov, A. Bykov, G. Molodij, V. Kalchenko, T. Jarvinen, A. Popov, K. Kordas, and I. Meglinski, J. Phys. D: Appl. Phys. 51, 155401 (2018). https://doi.org/10.1088/1361-6463/aab404

25. A. Sdobnov, A. Bykov, A. Popov, E. Zherebtsov, and I. Meglinski, Proc. SPIE 10685, 1068509 (2018). https://doi.org/10.1117/12.2306631

26. A. C. Volker, P. Zakharov, B. Weber, F. Buck, and F. Scheffold, Opt. Express 13, 9782 (2005). https://doi.org/10.1364/OPEX.13.009782

27. T. B. Rice, E. Kwan, C. K. Hayakawa, A. J. Durkin, B. Choi, and B. J. Tromberg, Biomed. Opt. Express 4, 2880 (2013). https://doi.org/10.1364/BOE.4.002880

28. D. A. Boas and A. K. Dunn, J. Biomed. Opt. 15, 011109 (2010). https://doi.org/10.1117/1.3285504

29. H. Cheng, Q. Luo, S. Zeng, S. Chen, J. Cen, and H. Gong, J. Biomed. Opt. 8, 559 (2003). https://doi.org/10.1117/1.1578089

30. J. Qiu, P. Li, W. Luo, H. Zhang, and Q. Luo, J. Biomed. Opt. 15, 016003 (2010). https://doi.org/10.1117/1.3290804

31. M. S. Wrobel, A. P. Popov, A. V. Bykov, M. Tuchin, and M. Jedrzejewska-Szczerskaa, Biomed. Opt. Express 7, 2088 (2016). https://doi.org/10.1364/BOE.7.002088

32. S. T. Flock, S. L. Jacques, B. C. Wilson, W. M. Star, and M. J. van Gemert, Laser. Surg. Med. 12, 510 (1992). https://doi.org/10.1002/lsm.1900120510

33. J. W. Pickering, S. A. Prahl, Van N. Wieringen, J. F. Beek, H. J. Sterenborg, and M. J. van Gemert, Appl. Opt. 32, 399 (1993). https://doi.org/10.1364/AO.32.000399

34. I. V. Meglinski and S. J. Matcher, Opt. Spectrosc. 91, 654 (2001). https://doi.org/10.1134/1.1412689

35. H. Soleimanzad, H. Gurden, and F. Pain, J. Biomed. Opt. 22, 010503 (2017). https://doi.org/10.1117/1.JBO.22.1.010503

36. J. H. Park, W. Sun, and M. Cui, Proc. Nat. Acad. Sci. U. S. A. 112, 9236 (2015). https://doi.org/10.1073/pnas.1505939112

37. J. He, H. Lu, R. Deng, L. Young, S. Tong, and X. Jia, EMBC Proc., 6971 (2015). https://doi.org/10.1109/EMBC.2015.7319996

38. A. K. Dunn, Ann. Biomed. Eng. 40, 367 (2012). https://doi.org/10.1007/s10439-011-0469-0

39. A. Y. Sdobnov, M. E. Darvin, E. A. Genina, A. N. Bashkatov, J. Lademann, and V. V. Tuchin, Spectrocim. Acta, A 197, 216 (2018). https://doi.org/10.1016/j.saa.2018.01.085

40. A. Y. Sdobnov, J. Lademann, M. E. Darvin, and V. V. Tuchin, Biochemistry (Moscow) 84, 144 (2019). https://doi.org/10.1134/S0006297919140098 